\title{
Universality and Halo Nuclei
}

\author{
Lauro Tomio ${ }^{1, a}$ \\ Instituto de Física Teórica, UNESP-Universidade Estadual Paulista, \\ Caixa Postal 70532-2, 01140-070, São Paulo, SP, Brazil
}

\begin{abstract}
Universal aspects of few-body systems will be reviewed motivated by recent interest in atomic and nuclear physics. The critical conditions for the existence of excited states in three-body systems with two-identical particles will be explored. In particular, we consider halo nuclei that can be modeled as three-body nuclear systems, with two halo neutrons and a core. In this context, we also discuss the low-energy neutron $-{ }^{19} \mathrm{C}$ elastic scattering, near the conditions for the appearance of an Efimov state.
\end{abstract}

\section{Introduction}

In order to understand the concept of universality in low-energy few-body systems and, in this context, describe low-energy properties of rich-neutron halo nuclei in a three-body model, we introduce here a historical review on some three-body properties and fewbody correlations which are well-known by the nuclear physicists. We point out that three-body low-energy properties have been quite well studied in the nuclear physics context since 1935 with Thomas [1], concerned with few-body aspects of nuclear physics, evidenced by some well-known correlations among low-energy nuclear-physics observables: Phillips line [2], Coester line [3] and Tjon line [4]. Such correlations will be discussed in section 2, together with analysis considering a few more recent works. In the same section, we also briefly discuss another relevant effect that was discovered by Efimov [5] in the nuclear physics context when considering low-energy three identical particles. This effect, which is also valid for more general three-body systems, will be further discussed along the other sections of the present review. As it will be pointed out [6], the Efimov effect is closely related to the results obtained by Thomas in [1].

Actually, a review on such few-body correlations is quite relevant in view of recent experiments in cold atom laboratories [7-11], which are claiming to have observed manifestations of the Efimov effect (for recent discussions on that, see also Refs. [12-14]). One should also realize that several low-energy few-body correlations, verified in few-nucleon systems, can be more deeply studied in atomic laboratories, considering the actual possibilities in varying the two-body interaction by using Feshbach resonance techniques [15].

Few-body correlations are also shown to be important to study properties of low-mass exotic nu-

\footnotetext{
${ }^{a}$ e-mail: tomio@ift.unesp.br
}

clei, with neutron halos, such as Helium-6, Lithium-11, Carbon-20, etc., which can be described as neutronneutron-core $(n-n-c)$ three-body systems [16-19]. In view of that, in section 3 , we will discuss universal aspects and scaling behavior of low-energy three-body systems, considering non-identical two kind of particles. The discussion is limited to the case of threeboson systems (with two different masses), in order to obtain threshold conditions for the existence of Efimov states. In this respect, in section 4, the approach is illustrated by considering the halo nucleus carbon$20\left({ }^{20} \mathrm{C}\right)$ in a three-body $n-n-{ }^{18} \mathrm{C}$ model. In this section, it is also reported scattering results for the $n-{ }^{19} \mathrm{C}$ system and an effective range analysis. The main conclusions and some perspectives are presented in the last section.

\section{Few-body correlations in nuclear physics}

\subsection{Thomas effect}

In 1935, when studying the fundamental forces binding the nucleons together, in a nonrelativistic quantum approach for the neutron-proton interaction and the triton structure, Thomas verified that the triton energy will collapse if the range of the two-body interaction goes to zero for a fixed two-body binding [1]. At the time this effect was recognized as a proof of the finite range of nuclear forces (See Sec. IV of Bethe and Bacher [20]) and also referred as Thomas's theorem [21], considering that Thomas was able to conclude that the range of the nuclear forces could not be less than $1.10^{-13} \mathrm{~cm}$ (On the range of nuclear forces and Thomas results, see also Refs. [22]). The collapse of the three-body ground-state for zero-range two-body interactions, sometimes also referred as Thomas's collapse, has been discussed in many other subsequent works: in the context of three-nucleon systems [23-28], 
as well as when discussing more general low-energy few-body properties and correlations [5,6,29-34]. It was pointed out by Gibson and Stephenson [26] that such collapsing effect can be understood by a qualitative simplified argument given by Wigner [35], relating the kinetic and potential energies in the deuteron and triton, when ignoring short-range repulsion and problem of saturation. From the Wigner's argument, in the deuteron one has a relation such that $2 T+V \approx 0$, while in the triton the corresponding relation is given by $3 T+2 V \approx-T$, which has no lower bound as the size of the system is decreased.

By considering that a three-boson system, interacting via two-body separable potentials with fixed two-boson binding, is known to lead to bound-state collapse (BSC) when the potential parameters allow two-boson S-matrix poles close to or on the real momentum axis [27], in Ref. [29] the BSC was investigated for the case of two-terms form-factor Tabakin potential [28]. In this case, after recognizing that such BSC is a manifestation of the Thomas collapse, the effect was shown to be accompanied by an increase in the average kinetic energy of the two-body bound state, which signals a decrease in the range of the twobody interaction for fixed two-body binding.

In the study of nuclear forces, several other correlations among observables have been found in the study of few-nucleon interactions $[2,4]$ and nuclear matter [3]. Later on, the Thomas effect was shown [6] to be closely related to another observed low-energy effect, the Efimov effect [5], which as discovered in 1970. This will be discussed in the next subsections.

\subsection{Phillips line}

One of the first general correlations verified in the nuclear force investigations, among low-energy observables, is known as the Phillips line [2]. Phillips, by analyzing several model calculations, found a consistent linear correlation between the triton binding energy and the doublet nucleon-deuteron scattering length [2]. An explanation of the Phillips line in the three-nucleon problem was presented by Efimov and Tkachenko in Ref. [36]. This line, which represents the correlation between calculated values of the triton binding energy and the neutron-deuteron doublet scattering length, is shown to be due to the fact that the binding energies of both the triton and the deuteron, as well as the energy eigenvalue of the two-nucleon singlet virtual state, are all small on the energy scale of nuclear forces. An equivalent statement is that the Phillips line is due to the loose spatial structure of these fewnucleon systems.

The observed strong correlation among theoretically calculated $s$-wave spin doublet neutron-deuteron scattering length and the triton binding energy, given by the Phillips line, was followed by several studies on the trinucleon observables [37-42]. Among them,

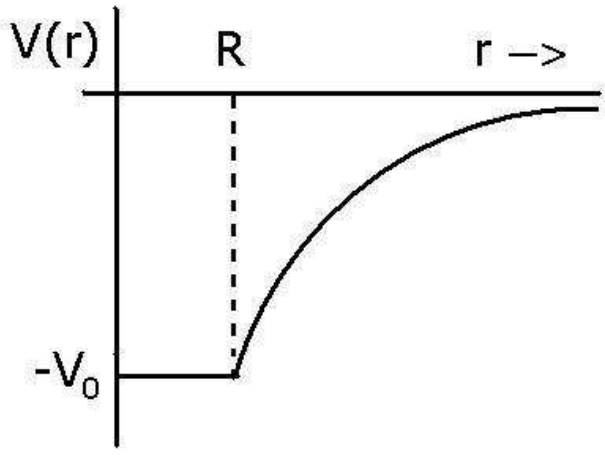

Fig. 1. Two-body potential model for the nucleondeuteron.

in the next, it will be considered the two-body analysis given in [42], from where more references can be traced on this subject, together with analysis of the Los Alamos group [39-41]. In Ref. [42], the trinucleon system was considered in a two-body model with Coulomb effect on bound and scattering states. As observed, the correlation of triton binding with the nucleon-deuteron scattering length can essentially be explained in a two-body model, by using the $N / D$ approach [38], or in a three-body model with zerorange nucleon-nucleon interaction. The aim in [42] was to suggest an effective nucleon-deuteron interaction in order to explain most of these properties. With the conjecture that the effective interaction is dominated by a truncated $r^{-2}$ interaction at large distances, it was demonstrated that the trinucleon lowenergy properties can be explained by varying only the short-range part of this effective interaction, keeping the long-range behavior unchanged. The depth $V_{0}$ of the potential is the required parameter. So, it was suggested the following effective interaction (see Fig. 1):

$$
V(r)=\left\{\begin{array}{ll}
-V_{0} & r<R \\
-V_{0}\left(\frac{R}{r}\right)^{2} e^{-\mu(r-R)} & r \geq R
\end{array},\right.
$$

where, in addition to $V_{0}$, we have also the parameters $\mu$ and $R$ which are given in [42].

First, the parameters are evaluated by requiring that the effective interaction produce the experimental values of the neutron-deuteron $(n-d)$ scattering length, ${ }^{2} a_{n d}$ and the binding energies, ${ }^{3} \mathrm{H}$ and ${ }^{3} \mathrm{He}$, where for the ${ }^{3} \mathrm{He}$ case it was added the Coulomb interaction. The parameters, with observables, are given in Table I of [42]. Next, the Phillips plot was easily reproduced by varying the short-range part $\left(V_{0}\right)$ of the effective interaction, as shown in Fig.2. The correlation of the triton binding with the $(s$-wave spin doublet) neutron-deuteron scattering length $\left({ }^{2} a_{n d}\right)$, as well as with the corresponding proton-deuteron scattering length $\left({ }^{2} a_{p d}\right)$, was explicitly demonstrated by 


\section{$19^{\text {th }}$ International IUPAP Conference on Few-Body Problems in Physics}

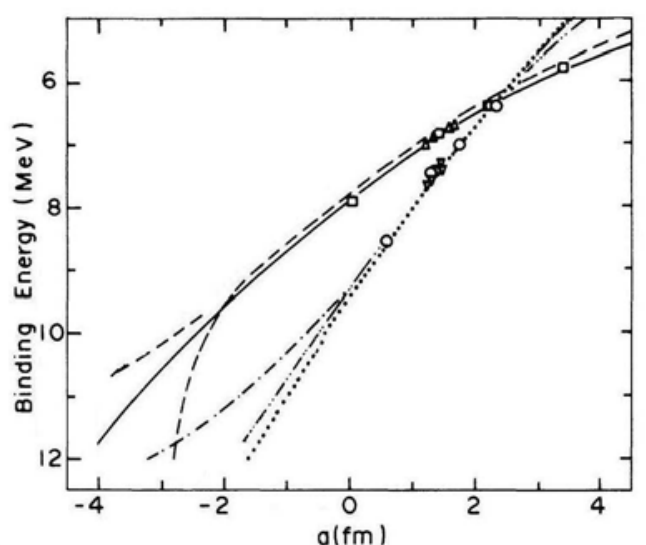

Fig. 2. Correlation between the trinucleon binding energy and the scattering length. From the results obtained in Ref. [42] with a two-body model we have the solid line for the proton-deuteron $(p-d)$ case; and the dotted line for the neutron-deuteron $(n-d)$ case. The other results shown in the figure are from Refs. [39] and [40]: the circles $(n-d)$ and squares $(p-d)$ are from [39]; triangles $(p-d)$ and inverted triangles $(n-d)$ from [40]. From [39,40] it is also shown the corresponding correlations for $p-d$ (dashedline) and $n-d$ (dashed-dotted and dashed-dotted-dotted lines).

using the same nucleon-deuteron effective interaction. Within this model, the predicted value for ${ }^{2} a_{p d}(\sim$ $0.15 \pm 0.1 \mathrm{fm})$ is consistent with the analysis of Refs. [39, 40]. (For the problem on the experimental determination of the proton-deuteron doublet scattering length, see Ref. [43].) In such two-body model, the $p-d$ polarization potential was neglected. However, it was found in [41] that, because of the very low polarizability of deuteron, the effect of the polarization potential on ${ }^{2} a_{p d}$ could be virtually neglected.

By considering this two-body model, it was possible to conclude that such a correlation is not a manifestation of three-body dynamics.

\subsection{Tjon line}

Following the above observed correlation of the triton binding with the neutron-deuteron doublet scattering length, Tjon observed a similar correlation between the three and four-body observables. More specifically, it was found that the ${ }^{4} \mathrm{He}$ energy is determined by the triton one, which is known as the Tjon line [4]. This correlation was also studied by Perne and Kröger [44] and by Noyes using nonrelativistic equations for three and four particle systems, within a zero-range scattering [45]. Once the triton energy is fitted to its experimental value the Tjon-line gives the observed ${ }^{4} \mathrm{He}$ binding. The observed correlation implies that, within the renormalization group approach [46], there is no need of a new scale to describe the four nucleon system. However, such result may be particular to the nuclear potential models used in the calculations, with their strong short-range repulsive interaction. In a more general context, the conclusion that the four-body scale is not independent of the three-body one has being discussed in Refs. [47,48]. As argued in [48] that it may be not valid near a Feshbach resonance in atomic systems, some experimental studies are being carried out in cold-atom latoratories [49], with actual analysis supporting the prediction that the universal tetramer states are in close connection with Efimov trimer [47].

However, one should note that a three-body repulsive force was introduced in [47] to stabilize the shallowest three-body state, against the variation of the cut-off. The three-body interaction may be attractive or repulsive with the attractive part indicating a possible independent behavior of the four body groundstate energy from the three-body one. In Ref. [48], it is shown that an independent four-body momentum scale, $\mu_{(4)}$, can drive the tetramer binding energy for fixed trimer energy and large scattering length $a$. The three- and four-body forces from the one-channel reduction of the atomic interaction near a Feshbach resonance is expected to disentangle the four- and threebody scales, $\mu_{(4)}$ and $\mu_{(3)}$, respectively. The four-body independent scale should be manifested through a family of Tjon-lines, with slope given by $\mu_{(4)} / \mu_{(3)}$ when $a^{-1}=0$. If this conjecture is realized, a new renormalization group limit cycle is expected to appear due to the new scale.

\subsection{Coester line}

By considering that for any given two-body Hamiltonian, there exists a large class of unitarily equivalent Hamiltonians that lead to the same scattering phase shifts at all energies, Coester, Day and Goodman [3] have studied the saturation curves for reasonable equivalent potentials. It was observed that the saturation minimum shifts to higher or lower density as the binding increases or decreases. Softening the potential increases the binding.

This interesting correlation was recently studied in Ref. [50], in a search for possible generalizations of the Tjon line to the many-nucleon context, from light nuclei to nuclear matter. As the nuclear matter saturates due to the composed repulsive and attractive short-ranged two-nucleon potential, it may also seen as a typical low-energy problem. So, it is natural to search for possible connections between the proper few-body scales with those of many-body problem. It was pointed out evidences for scaling between light nuclei binding energies and the triton, and argued that the saturation energy and density of nuclear matter are correlated to the triton binding energy, $B_{t}$. The available systematic nuclear matter calculations indicates a possible band structure representing these correlations. It was found that the original correlation between the nuclear matter binding energy per nucleon, with the Fermi momentum described by the 


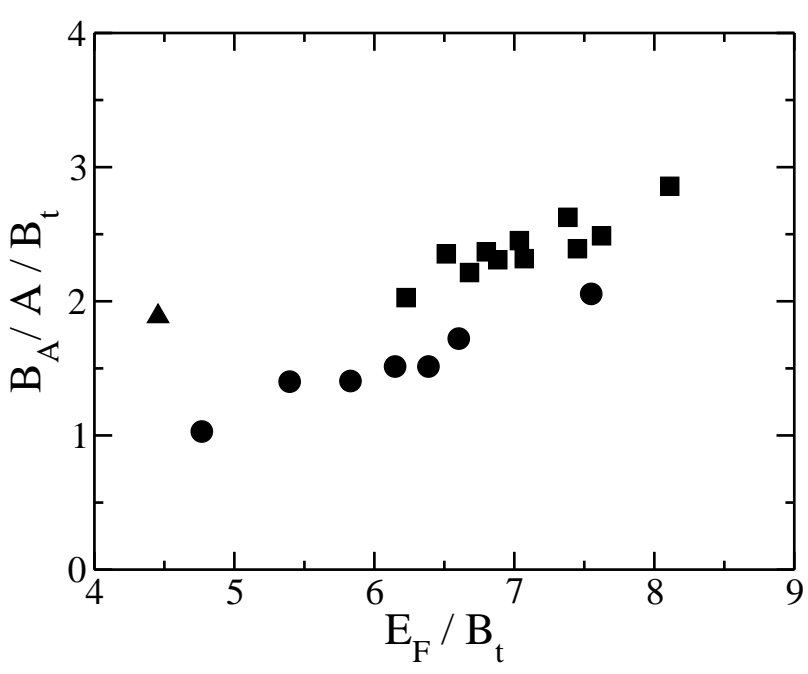

Fig. 3. Infinite nuclear matter binding energy as a function of $E_{F}$, both in units of the triton binding energy. The calculation results are extracted from Ref. [51] (solid circles and squares). The squares includes the single particle contribution in the continuum. The full triangle represents the empirical values. This figure was reproduced from Ref. [50].

Coester band, can be seen as robustly represented by the scaling of nuclear matter properties with the triton binding energy. As an example, in Fig. 3 we reproduce one of the results shown in [50], where we can see the behavior of the finite nuclear matter binding energy $B_{A} / A$ (where $A$ is the mass number) as a function of the Fermi energy $E_{F}$, both given in units of $B_{t}$.

\subsection{Efimov effect}

The Efimov effect [5] refers to the infinite number of $s$-wave three-body energy levels, which appears when a two-body state is exactly at the dissociation threshold. So, the number of three-body states start to increase as one reduces to zero the absolute value of the two-body binding (virtual) energy; or, correspondingly, when one increases the absolute value of the two-body scattering length to infinite. As verified, three bosons can form a loosely bound system even if the two-particle attraction is too weak to allow two bosons to form a pair.

This apparently counter-intuitive effect is shown in Ref. [6] to be related to the Thomas effect by a scaling transformation. Both, Efimov and Thomas effects, result from the same singularity structure of the kernel of the nonrelativistic scattering integral equation of a three-body system in the $s$-wave. It was also verified that both effects occur in three dimensions but not in two dimensions. More precisely, it was also demonstrated by Jensen, Riisager and Fedorov [33] that the Thomas and Efimov effects can only occur for dimensions between 2.3 and 3.8. A simple variational proof of the Thomas effect, provided in Ref. [30], shows clearly why it is related to the Efimov effect. The relation of both effects was also recognized in some other recent works, such as in Refs. [14,52].

The experimental study of Efimov states became accessible in ultra-cold atom laboratories, in view of the possibilities to alter the two-body scattering length, in a wide range by using Feshbach resonance techniques [15]. In 2006, it was reported in Ref. [8] that they had detected Efimov states in scattering using caesium atoms at 10 nanokelvin. This result was also discussed by Lee et al. [14]. More recent reports on experimental investigations on the existence of Efimov spectrum in ultracold atoms are given in Refs. [10,11].

\section{Universality and Scaling}

The concepts of universality, scaling, renormalization, and limit cycle have been considered in many recent works, in order to understand and classify the lowenergy few-body correlations. The relevance of such studies can be appreciated in view of the recent experiments $[7,8,10,11]$ that are confirming universal properties coming from the Efimov physics [5] occurring for large two-body scattering lengths [32-34,12]. Near a Feshbach resonance the two-atom scattering length, $a$, can vary from very large negative values to positive values, allowing virtual or weakly-bound dimers. The scattering length is large in respect to the atom-atom interaction range $\left(r_{0}\right)$, driving to the use of concepts developed for short range interactions and halo states [33]. In the limit of large $a$, the interaction can be taken as of zero range [53]. The appearance of Thomas-Efimov states in three-boson systems is controlled by the ratio $r_{0} /|a| \rightarrow 0$. In this exact limit, it is observed an infinite sequence of three-body bound states $[18,54]$, identified [55] with an underlying renormalization group limit cycle [46]. The collapse of the three-boson system when the two-body interaction range goes to zero for fixed $a$ demands one three-body scale to stabilize the system.

Such sequence of Efimov states, appearing as the two-body subsystem energy is reduced to zero, was presented in [54] as a scaling function when considering the interaction of three particles system $\alpha-\alpha-\beta$, with the corresponding masses given by $M_{\alpha}$ and $M_{\beta}$ having the mass-ratio $A \equiv M_{\beta} / M_{\alpha}$. The scaling function is given by the ratio of two consecutive three-body energy states $B_{3}^{N}$ and $B_{3}^{N+1}$ (the ground-state correspond to $N=0)$ :

$$
\frac{B_{3}^{N+1}}{B_{3}^{N}}=\mathcal{F}\left(\frac{K_{\alpha \alpha}}{\sqrt{B_{3}^{N}}}, \frac{K_{\alpha \beta}}{\sqrt{B_{3}^{N}}} ; A\right),
$$

where, for $\gamma \equiv \alpha, \beta, K_{\alpha \gamma}$ is related to the two-body scattering length $a_{\alpha \gamma}$, or the two-body energy $B_{\alpha \gamma}$ :

$$
K_{\alpha \gamma} \equiv \frac{1}{a_{\alpha \gamma} \sqrt{2 \mu_{\alpha \gamma}}}= \pm \sqrt{B_{\alpha \gamma}}
$$

In the above, the signal $(+)$ stands for a bound twobody state, and (-) for a virtual two-body state; with 


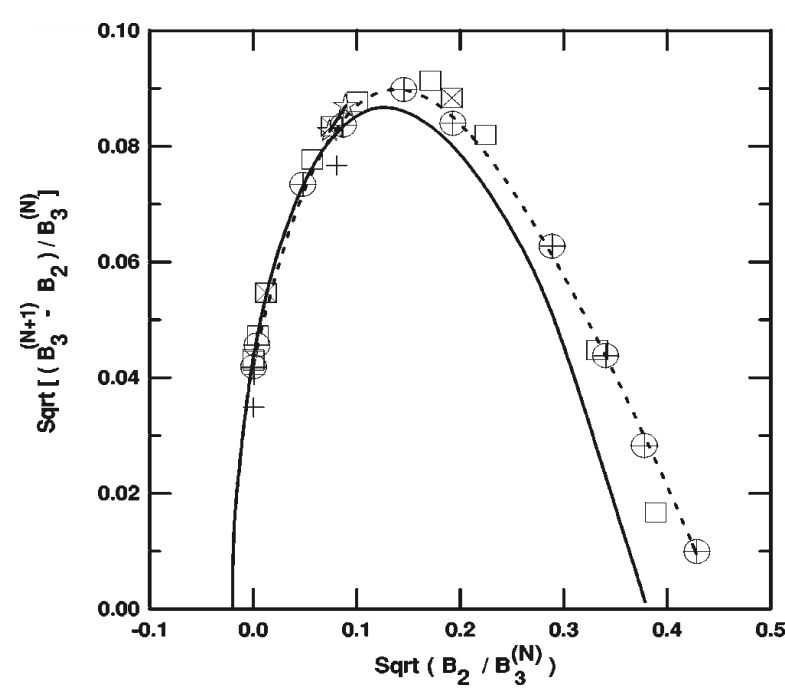

Fig. 4. Scaling limit (solid line) compared with realistic model calculations for the ${ }^{4} \mathrm{He}$ trimer, as shown in [54]. With dashed, we have a guide line through the realistic model calculations.

$\mu_{\alpha \gamma}$ the reduced mass for the $(\alpha \gamma)$ system. We should observe that, the exact Efimov limit is given when the two-body energy states are equal to zero. This scaling limit, by using the renormalized zero-range approach, was first presented in Fig.2 of Ref. [54], considering three identical particles. In Fig. 4 we reproduce this scaling function, compared with realistic model calculations for the ${ }^{4} \mathrm{He}$ trimer. From such comparison with finite range results, one can estimate range effects, which are enhanced as the two-body binding energy is increased.

More recently, this results for the scaling function were further explored in order to verify the behavior of the three-body states. In figure 5 (where $E_{2,3} \equiv-B_{2,3}$ in case of bound-states), it is shown the Efimov states following a continuous transition when we vary the two-body scattering length $a$. A virtual state emerges by the elastic scattering cut coming from the second Riemmann sheet becoming a bound state. Then, for a virtual two-body state $(a<0)$ a bound state turns into a resonance. For $a<0$ we have a more favorable condition for the formation of trimers as the competition with weakly bound dimers is absent.

By considering the more general case of $\alpha-\alpha-\beta$ system, it was determined the region where at least one Efimov excited state can exist in a parametric region defined by the ratios of the energies of the subsystems, in units of the three-body ground-state energy. In this case, it was verified the possible existence of Efimov states in a few light nuclei with two-neutron halos, which are considered as three-body neutron-neutron-core $(n-n-c)$ systems, such that

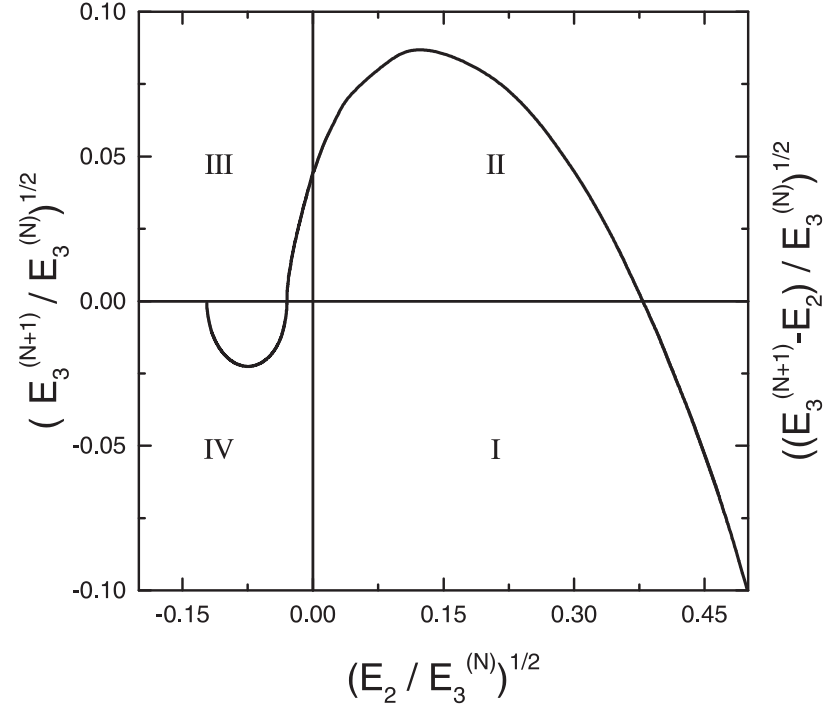

Fig. 5. Ratio of the $(\mathrm{N}+1)^{t h}$ trimer binding, virtual or resonance energy, $E_{3}^{(N+1)}$, with respect to the threshold ( $E_{2}$ is the two-body binding energy) and the $\mathrm{N}^{\text {th }}$ trimer binding energy, $E_{3}^{(N)}$ as a function of the dimer energy $E_{2}$ and $E_{3}^{(N)}$. Regions I and II refer, respectively, to virtual and bound trimers for bound dimers. Regions III and IV refer, respectively, to bound states and resonances for virtual dimers. All results are for $N=0$. The right vertical label is valid for regions I and II and the left one for regions III and IV.

$\alpha \equiv n$ and $\beta \equiv c$. The result is shown in Fig. 6 . From the experimental data that were examined, only the carbon-20 was found with possibility to have at least one excited Efimov state. In Fig. 6 it is also shown the analytical cut structure of each four regions of the plane. The borromean case, where all two-body subsystems are unbound, is labelled as region (III). As discussed in Ref. [56] for the trajectories of excited three-body Efimov states, when at least one two-body substystem is bound, the Efimov bound state turns to a virtual state when decreasing the corresponding scattering length. However, in the Borromean case, such three-body bound state turns to a resonance.

The above results, shown in Figs. 4 and 6, are combined in a pictorial 3D scaling plot, given in Fig 7. For the case that $E_{n n}=E_{n c}=0$ we have the exact Efimov limit, with $z=\sqrt{\left(E_{3}^{(N+1)}\right) / E_{3}^{(N)}}=\sqrt{1 / 515}$. Finally, we should also observe that the universal properties of few-body physics, renormalization and scaling limits, have also being studied by several authors in the context of Effective Field Theories (EFT). For some reviews and recent works on EFT applied to few-nucleon systems, see Refs. $[32,57,58]$ and references therein. 


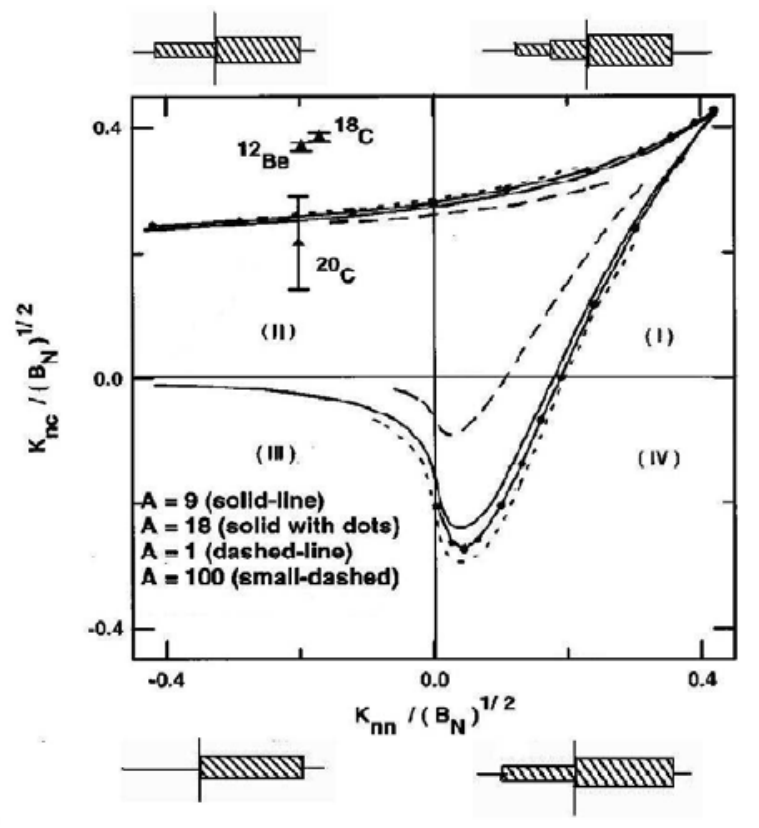

Fig. 6. Parametric region defined by $K_{n n} / \sqrt{B_{N}}$ and $K_{n c} / \sqrt{B_{N}}$. At the boundaries, for different mass ratios $A$, the binding energy of the $(N+1)$ th Efimov state is zero. For details, on the comparison with a few experimental halo-nuclei systems, see Ref. [18]. The analytical two- and three-body cut structures are schematically represented near the corresponding region, in the upper and lower side of the figure.

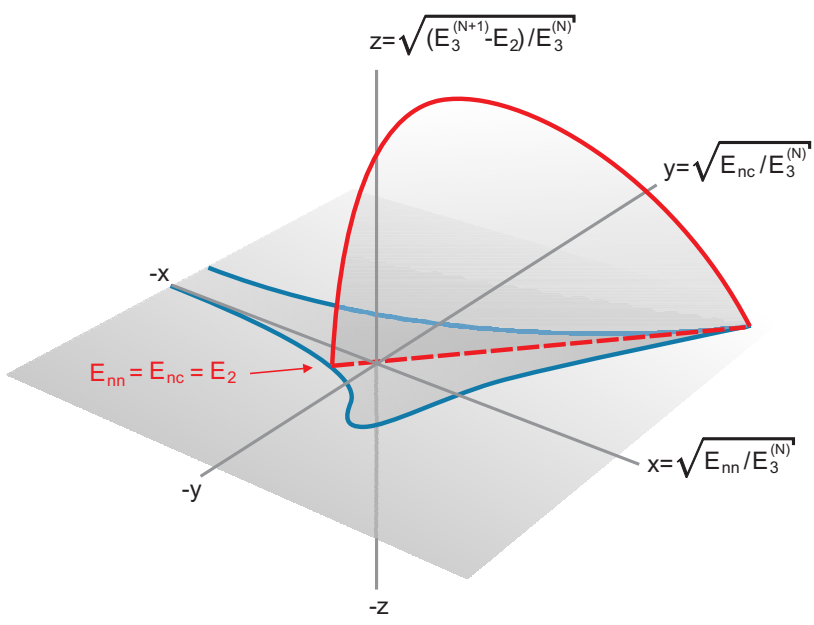

Fig. 7. Threshold conditions and scaling function. When $x=y=0$ we have the Efimov result $z=\sqrt{1 / 515} \approx 0.044$.

\section{Halo nuclei - Efimov states and scattering properties}

The actual renewed interest in investigations of Efimov states brought by the possibilities in atomic laboratories, commented in the previous sections, had also a lot of interest in nuclear context with the studies on the properties of exotic nuclei systems with two halo neutrons $(n-n)$ and a core $(c)$ [33]. In this respect, some of the most favorable systems for search- ing Efimov states in systems with two-neutron halos are light nuclei such as Helium-6 $\left({ }^{6} \mathrm{He}\right)$, Lithium$11\left({ }^{11} \mathrm{Li}\right)$, Beryllium-14 $\left({ }^{14} \mathrm{Be}\right)$, Carbon-18 $\left({ }^{18} \mathrm{C}\right)$, and Carbon-20 $\left({ }^{20} \mathrm{C}\right)$. Among these systems, as verified in Fig. 6, one of the possible candidates to present these states is the ${ }^{20} \mathrm{C}[18,19,33,59]$.

\subsection{Radii of halo nuclei systems}

For a general classification scheme of A-A-B systems, applied not only for halo nuclei, but also to weakly bound molecules as well, see Ref. [60], where it is made a systematic study of the root-mean-square distances between the constituents of weakly-bound nuclei systems, $\sqrt{\left\langle r_{n A}^{2}\right\rangle}$ and $\sqrt{\left\langle r_{n n}^{2}\right\rangle}$. The study was focused on the halo nuclei ${ }^{11} \mathrm{Li}$ and ${ }^{14} \mathrm{Be}$. The approach considered was a renormalized three-body model with pairwise Dirac- $\delta$ interactions, with a minimal number of physical inputs directly related to observables, such as the two-neutron separation energy and the n-n and n-core s-wave scattering lengths. As it was concluded, the low-energy properties of the halo neutrons are, to a large extend, model independent as long as few physical input scales are fixed. The results are consistent with experimental data, within the error bars. A systematic underestimation of the data was observed, which are naturally due to the simplified model that was considered, where some properties are missing, such as the finite size of the core allied to the antisymmetrization of the total nuclear wave function.

The consequences of a classification scheme proposed in Ref. [61] for weakly-bound three-body systems was studied in detail in [60], by analyzing the dimensionless products $\sqrt{\left\langle r_{n A}^{2}\right\rangle E_{3}}$ and $\sqrt{\left\langle r_{n n}^{2}\right\rangle E_{3}}$ in terms of scaling functions depending on dimensionless products of scattering lengths and square-root of the neutron-neutron separation energy. The qualitative properties of the different possibilities of threebody systems are understood in terms of the effective attraction in the model: when a pair has a virtual state the effective interaction is weaker than when the pair is bound. Thus, a three-body system has to shrink to keep the binding energy unchanged if a pair which is bound turns to be virtual. Several examples illustrate this property, which show that dimensionless sizes, $\sqrt{\left\langle r_{n A}^{2}\right\rangle E_{3}}$ and $\sqrt{\left\langle r_{n n}^{2}\right\rangle E_{3}}$, increase from Borromean (unbound subsystems), Tango (two unbound subsystem), Samba (one unbound subsystem) and to AllBound configurations. And the size is expected to increase beyond limits when a nonvanishing three-body energy hits the scattering threshold, with the Borromean configuration being the only exception.

\subsection{Scattering properties and the ${ }^{20} \mathrm{C}$}

When studying the scattering properties of the halo nuclei ${ }^{20} \mathrm{C}$, in a three-body model $n-n-{ }^{18} \mathrm{C}$, the authors of Refs. [59] have observed a strong enhancement 


\section{$19^{\text {th }}$ International IUPAP Conference on Few-Body Problems in Physics}

of the $n-{ }^{19} \mathrm{C}$ elastic cross section. The result of this kind of study can be quite relevant also for the analysis of actual cold-atom experimental results, where the two-body interaction can be varied. In this respect, the collisional properties of weakly-bound dimers with mixture of two-species of atoms, have recently been considered in Ref. [62]. The proximity of an Efimov state (bound or virtual) makes the elastic cross-section extremely sensitive to the corresponding pole of the $S$ matrix. For the analysis of the $n-n-{ }^{18} \mathrm{C}$, one should first observe that this is not a Borromean system, considering that it has only one subsystems unbound. The analytic properties of the S-matrix are expected to be quite different for Borromean system in comparison with systems where some (or all) of the subsystems are bound. In case of Borromean systems, only the three-body continuum exists, while in the other cases the two-body continuum also appears.

Considering the above, as well as the recent motivations on the behavior of Efimov states, going from excited bound states to virtual or resonant states as the two-body scattering length is altered, it was investigated in Ref. [56] the trajectory of the first excited Efimov state using a renormalized zero-range threebody model for a system with two bound and one virtual two-body subsystems. Previously, it was already investigated in Ref. [63] the occurrence of $p$-wave virtual states in three-body non-Borromean halo nuclei such as $n-n-{ }^{18} \mathrm{C}$. By considering the analytical cut structure of this kind of three-body systems, it was shown [56] for the trajectories of excited three-body Efimov states that, when at least one two-body subsystem is bound, the Efimov bound state turns to a virtual state when decreasing the corresponding scattering length. However, in the Borromean case, such three-body bound state turns to a resonance. The analytical cut structures of the four possible regions are also schematically represented in Fig. 6, near the corresponding region. The Borromean case, where all two-body subsystems are unbound, is labelled as region (III); in all the other three regions we have to consider at least one bound subsystem.

Following the studies on the trajectories of Efimov excited three-body states $[56]$, the ${ }^{20} \mathrm{C}$ scattering properties were further explored in Ref. [64] by analysing the elastic scattering of a neutron on ${ }^{19} \mathrm{C}$ near the condition for an Efimov state of ${ }^{20} \mathrm{C}$. The $n-n-{ }^{18} \mathrm{C}$ halo system presents a virtual state that turns into an excited state when the ${ }^{19} \mathrm{C}$ binding is decreased [56]. So, close to this condition, it is suggestive to study the low-energy $n-{ }^{19} \mathrm{C}$ elastic scattering in $s$-wave as the Efimov poles of the scattering amplitude are near the elastic scattering threshold. By using a zero-range interaction, it was shown in [64] that the real part of the elastic $s$-wave phase-shift $\left(\delta_{0}^{R}\right)$ presents a zero, or a pole in $k \cot \delta_{0}^{R}$, when the system has an Efimov excited or virtual state. More precisely the pole scales with the energy of the Efimov state (bound or virtual).
In view of the structure of the scattering $S$-matrix for the $n-{ }^{19} \mathrm{C}$ system, and corresponding similarity with the neutron-deuteron $(n-d)$ system, one should recall the long-time discussion on the $n-d$ elastic doublet state scattering, studied in several authors [6571]. The change in the off-shell behavior of the twobody potential, or three-body forces, modifies the corresponding phase-shift correlated to the triton binding in a way that the scattering length can vanish. This was already seen in the well-known Phillips plot where the doublet scattering length is presented as a function of the triton binding energy [2]. In the case of zero scattering length, $k \cot \delta_{0}$ has a pole at zero relative $n-d$ kinetic energy, pointed out in the analysis of the experimental data for the $n-d$ doublet $s$-wave phase-shifts $[65,66]$. Van Oers and Seagrave [66] proposed to incorporate a pole in a phenomenological effective range formula used to fit the $k \cot \delta_{0}$ low energy data for the $n-d s$-wave doublet state just below the elastic threshold. The effective range expansion has a form given by

$$
k \cot \delta_{0}=-A+B k^{2}-\frac{C}{1+D k^{2}},
$$

where $A, B, C$, and $D$ are fitted constants. The existence of the triton virtual state was found on the basis of the effective range expansion [69]. From the solution of a three-body model with separable two-body interactions, it was also suggested that the triton virtual state appears from an excited Efimov state moving to the non-physical energy sheet through the elastic cut [72]. The same reasoning can be applied to the case of an excited Efimov state of the $n-n-{ }^{18} \mathrm{C}$ system, where the pole of the S-matrix migrates to the second energy sheet through the elastic $n-{ }^{19} \mathrm{C}$ cut when the binding energy of the neutron in ${ }^{19} \mathrm{C}$ is increased.

In [64] it was shown that the $n-{ }^{19} \mathrm{C} s$-wave lowenergy phase-shift exhibit analytical properties similar to the one found in the case of the $n-d$ doublet state, where existence of a low-energy pole in $k \cot \delta_{0}^{R}$ was established. By extending the analysis, it is observed that the physics related to the Efimov effect is also implying a zero in the atom-dimer scattering length, and consequently a pole in the effective range expansion at zero kinetic energy. A pole in the effective range expansion of the $n-{ }^{19} \mathrm{C}$ elastic phase-shift appears in a quite good qualitative agreement with the above analysis. The parametrization of the phase-shift was done by a simple analytical formula of the effective range expansion with a pole, as proposed long ago by Van Oers and Seagrave[66] to fit the low-energy experimental data of the doublet s-wave neutron-deuteron phase-shift. In this case, the low-energy parametrization of the effective range expansion is given by:

$$
k \cot \delta_{0}^{R}=\frac{-a_{n-19 C}^{-1}+\beta E+\gamma E^{2}}{1-E / E_{0}},
$$

where $a_{n-19} C$ is the $n-{ }^{19} C$ scattering length, with $\beta$ and $\gamma$ the effective range parameters to be adjusted. 
EPJ Web of Conferences

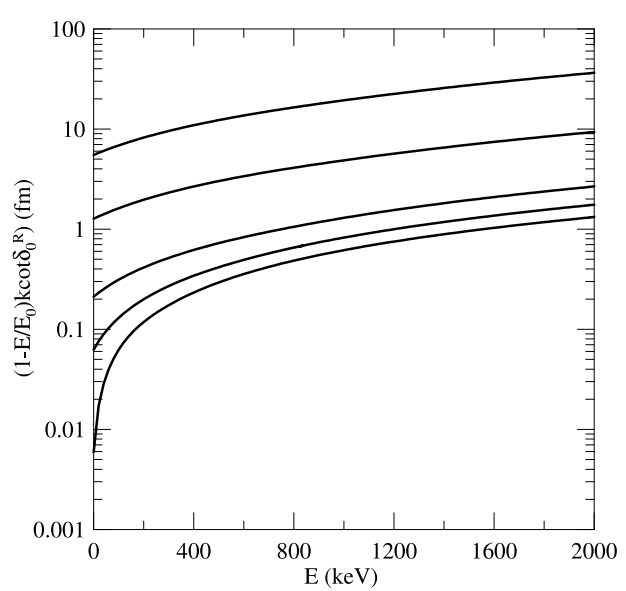

Fig. 8. $\left(1-E / E_{0}\right) k \cot \delta_{0}^{R}$ as a function of the center-ofmass kinetic energy. From bottom to top the curves corresponds to the following ${ }^{19} \mathrm{C}$ energies: $200,400,600,800$ and $850 \mathrm{keV}$. Reproduced from Ref. [64].

$E_{0}$ is the position of the pole with respect to the threshold for elastic scattering.

The numerical solutions for $\left(1-E / E_{0}\right) k \cot \delta_{0}^{R}$ as a function of the CM kinetic energy are shown in Fig. 8, for different values of $\left|E_{19} \mathrm{C}\right|$ between 200 and $850 \mathrm{keV}$. The effective range parameters are given in Table 1 , obtained from a fit of (5) to the results shown in Fig.8.

Table 1. Effective range parameters in Eq. (5) for different $\left|E_{19} \mathrm{C}\right|$ energies, as determined in Ref. [64].

\begin{tabular}{|ccccc|}
\hline $\begin{array}{c}\left|E_{19 \mathrm{C}}\right| \\
(\mathrm{keV})\end{array}$ & $\begin{array}{c}a_{n-19 \mathrm{C}} \\
(\mathrm{fm})\end{array}$ & $\begin{array}{c}\beta \\
(\mathrm{fm} . \mathrm{keV})^{-1}\end{array}$ & $\begin{array}{c}\gamma \\
\left(\mathrm{fm} . \mathrm{keV}^{2}\right)^{-1}\end{array}$ & $\begin{array}{c}E_{0} \\
(\mathrm{keV})\end{array}$ \\
\hline 200 & -169.2 & $5.6910^{-4}$ & $4.6710^{-8}$ & 1442.7 \\
400 & -16.03 & $6.7410^{-4}$ & $8.8210^{-8}$ & 823.9 \\
600 & -4.721 & $9.3410^{-4}$ & $1.4610^{-7}$ & 451.4 \\
800 & -0.789 & $3.1110^{-3}$ & $4.4210^{-7}$ & 115.0 \\
850 & -0.181 & $1.2010^{-2}$ & $1.6410^{-6}$ & 28.8 \\
\hline
\end{tabular}

\section{Summary with perspectives}

In conclusion, we start by reviewing some low-energy few-nucleon correlations that are quite well-known by the nuclear physicists, such as the Thomas collapse of the three-body ground state when the two-body range interaction goes to zero, and the Phillips line, which gives a correlation between values of the triton binding energy and the neutron-deuteron doublet scattering length. Some other few-body correlations were discussed in this context, such as the Tjon and Coester lines. These correlations among few-nucleon observables was followed by a discussion on the Efimov effect, represented by the accumulation of infinite three-body energy levels in the limit when the inverse of the two-body scattering length is zero. All the above revisions were considered in the actual context, together with some recent related works.

A discussion on universality and scaling applied to low-energy few-body systems, which is present in the physics related to the Efimov effect, is given in section 3. It is pointed out that the relevance of such low-energy few-body correlations are due to the actual possibilities to be verified in cold atom experiments, where the two-body interaction can be varied by using Feshbach resonance techniques.

Next, in section 4, it was discussed light halo-nuclei properties, considering the size of such three-body systems, trajectories of Efimov states and scattering properties. For the scattering properties, the study was exemplified with the Carbon-20, in view of recent calculations done for the $n-{ }^{19} \mathrm{C}$ elastic cross section and phase-shift analysis. In this context, some old studies on the neutron-deuteron properties was revised, considering the similiarity of such system with $n-{ }^{19} \mathrm{C}$.

Finally, it is relevant to emphasize the rich perspectives opened by the recent experimental researches with few-atom and molecular systems in ultracold laboratories, where previous correlations first verified in nuclear physics can now be tested in atomic and molecular physics. The low-energy few-body correlations can be studied by also considering different kind of particles, from Bosons to Fermions. As observed, within the renormalization group approach, the observed correlation are governed by some physical scales. As one increases the number of interacting particles, one can also study the possible necessity of independent new scales to describe the corresponding observables. Actually, this is being done in the context of four interacting particles.

The improvement observed in the techniques used in the coldatom laboratories has also opened many new theoretical possibilities to study observable correlations in few-particle systems, such that one can explore the limits of validity of such correlations by introducing new constraints (varying the external traps, Coulomb effects, etc.). In this respect, in the perspectives that mixed atom-dimer phases near the Efimov limit can be tested experimentally, we should mention a few works [73] in the context of atomic condensates near the scattering threshold.

\section{Acknowledgements}

My thanks to Tobias Frederico, Antonio Delfino, Marcelo Yamashita, Varése Timóteo, for discussions and collaborations reported here. Work partially supported by the Brazilian agencies Fundação de Amparo à Pesquisa do Estado de São Paulo (FAPESP) and Conselho Nacional de Desenvolvimento Científico e Tenológico (CNPq).

\section{References}

1. L. H. Thomas, Phys. Rev. 47, 903 (1935). 
$19^{\text {th }}$ International IUPAP Conference on Few-Body Problems in Physics

2. A. C. Phillips, Nucl. Phys. A 107, 209 (1968); Rep. Prog. Phys. 40, 905 (1977).

3. F. Coester, B. Day, and A. Goodman, Phys. Rev. C 1, 769 (1970) and Phys. Rev. C 5, 1135 (1972).

4. J.A. Tjon, Phys. Lett. B 56, 217 (1975).

5. V. Efimov, Phys. Lett. B 33, 563 (1970).

6. S. K. Adhikari, A. Delfino, T. Frederico, I. D. Goldman, and L. Tomio, Phys. Rev. A 37, 3666 (1988).

7. T. Weber et al., Phys. Rev, Lett. 91, 123201 (2003).

8. T. Kraemer et al., Nature 440, 315 (2006).

9. S. Knoop, et al., Nature Physics 5, 227 (2009).

10. M. Zaccanti, et al., Nature Physics 5, 586 (2009).

11. G. Barontini, et al., Phys. Rev. Lett. 103, 073202 (2009).

12. E. Braaten and H.-W. Hammer, Ann. of Phys. 322, 120 (2007).

13. I. Baccarelli, et al., Phys. Rep. 452, 1 (2007).

14. M. D. Lee, T Köhler and P. S. Julienne, Phys. Rev. A 76, 012720 (2007).

15. E. Timmermans, P. Tommasini, M. Hussein, A. Kerman, Phys. Rep. 315, 199 (1999).

16. D.V. Fedorov, A.S. Jensen, and K. Riisager, Phys. Rev. Lett. 73, 2817 (1994).

17. P. G. Hansen, A. S. Jensen, B. Jonson, Annu. Rev. Nucl. Part. Sci. 45, 591 (1995).

18. A. E. Amorim, T. Frederico, and L. Tomio, Phys. Rev. C56, R2378 (1997).

19. I. Mazumdar and V. S. Bhasin, Phys. Rev. C 56, R5 (1997).

20. H. A. Bethe and R. F. Bacher, Rev. Mod. Phys. 8, $82(1936)$.

21. R. L. Hall, Proc. Phys. Soc. 91, 787 (1967).

22. R. L. Pease and H. Feshbach, 88, 945 (1952); H. Feshbach and S. I. Rubinow, Phys. Rev. 98, 188 (1955).

23. L. M. Delves and A. C. Phillips, Rev. Mod. Phys. 41, 497 (1969).

24. J. S. C. McKee, Rep. Prog. Phys. 33, 691 (1970).

25. H. P. Noyes,Phys. Rev. Lett. 25, 321 (1970).

26. B. F. Gibson and G. J. Stephenson, Phys. Rev. C 8, 1222 (1973).

27. J. E. Beam, Phys. Lett. 30B, 76 (1969); V. A. Alexxandrini and C. A. Garcia Canal, Nucl. Phys. A 133, 950 (1969); G. Pantis, H. Fiedeldey, and D. W. L. Sprung, Z. Phys. A 291, 367 (1979); G. Rupp, L. Streit, and J. A. Tjon, Phys. Rev. C 31, 2285 (1985).

28. F. Tabakin, Phys. Rev. 174, 1208 (1968).

29. A. Delfino, S. K. Adhikari, and L. Tomio, Phys. Rev. C 46, 471 (1992).

30. F. A. B. Coutinho, J. F. Perez, and W. F. Wreszinski, J. Math. Phys. 36, 1625 (1995).

31. A. Delfino, T. Frederico, and L. Tomio, Few-Body Syst. 28, 259 (1992).

32. P. F. Bedaque and U. van Kolck, Ann. Rev. Nucl. Part. Sci. 52, 339 (2002).

33. A. S. Jensen, K. Riisager, D. V. Fedorov, and E. Garrido Rev. Mod. Phys. 76, 215 (2004).
34. E. Braaten and H.-W. Hammer, Phys. Rep. 428, 259 (2006).

35. E. Wigner, Phys. Rev. 43, 252 (1933).

36. V. E. Efimov and Tkachenko, Phys. Lett. 157B, 108 (1985).

37. H. Zankel and L. Mathelitsch, Phys. Lett. 132B 27 (1983); G.H. Berthold and H. Zankel, ibid. 162B, 208 (1985); G.H. Berthold and H. Zankel, Phys. Rev. C 34, 1203 (1986).

38. S.K. Adhikari and J.R.A. Torreão, Phys. Lett. 132B, 257 (1983).

39. J.L Friar, B.F. Gibson, G.L. Payne, and C.R. Chen, Phys. Rev. C 30, 1121 (1984).

40. C.R. Chen, G.L. Payne, J.L Friar, and B.F. Gibson, Phys. Rev. C 33, 401 (1986).

41. Gy. Bencze, C. Chandler, J. L. Friar, A. G. Gibson and G. L. Payne, Phys. Rev. C 35, 1188 (1987).

42. L. Tomio, A. Delfino, and S.K. Adhikari, Phys. Rev. C 35, 441 (1987).

43. T. C. Black, H. J. Karwowski, E. J. Ludwig, A. Kievsky, S. Rosati and M. Viviani, Phys. Lett. B 471, 103 (1999).

44. R. Perne and H. Kröger, Phys. Rev. C 20, 340 (1979).

45. H. P. Noyes, Phys. Rev. C 26, 1858 (1982).

46. K.G. Wilson, Phys. Rev. D3, 1818 (1971).

47. L. Platter, H.-W. Hammer and U.-G. Meissner, Phys. Lett. B 607, 254 (2005); H.-W. Hammer and L. Platter, Eur. Phys. J. A 32, 113 (2007).

48. M. Yamashita, L. Tomio, A. Delfino, and T. Frederico, Europhys. Lett. 75, 555 (2006).

49. F. Ferlaino, et al., Phys. Rev. Lett. 102, 140401 (2009); F. Ferlaino, et al., Phys. Rev. Lett. 101, 023201 (2008).

50. A. Delfino, T. Frederico, V.S. Timóteo, and L.Tomio, Phys. Lett. B 634, 185 (2006).

51. R. Machleidt, Adv. Nucl. Phys. 19, 189 (1989).

52. B.D. Esry and C.H. Greene, Nature 440, 289 (2006).

53. R. Jackiw, in M. A. B. Beg Memorial Volume, edited by A. Ali and P. Hoodbhoy (World Scientific, Singapore, 1991).

54. T. Frederico, L. Tomio, A. Delfino, A. E. A. Amorim, Phys. Rev. A60, R9 (1999).

55. R.F. Mohr, R.J. Furnstahl, H.-W. Hammer, R.J. Perry, and K.G. Wilson, Ann. Phys. 321, 225 (2006).

56. M. T. Yamashita, T. Frederico and L. Tomio, Phys. Lett. B 660,339 (2008); Phys. Rev. Lett. 99, 269201 (2007).

57. E. Epelbaum, H.-W. Hammer, and Ulf-G. Meissner, Rev. Mod. Phys. 81, 1773 (2009).

58. C.A. Bertulani, H.-W. Hammer, and U. van Kolck, Nucl. Phys. A 712, 37 (2002); D. L. Canham and H.-W. Hammer, Eur. Phys. J. A 37, 367 (2008); E. Epelbaum and J. Gegelia, Eur. Phys. J. A 41, 341 (2009).

59. V. Arora, I. Mazumdar and V. S. Bhasin, Phys. Rev. C 69, 061301(R) (2004); I. Mazumdar, A.R.P. Rau, V.S. Bhasin, Phys. Rev. Lett. 97, 
$062503(2006)$.

60. M. T. Yamashita, T. Frederico, and L. Tomio, Nucl. Phys. A 735, 40 (2004).

61. A.S. Jensen, K. Riisager, D.V. Fedorov and E. Garrido, Europhys. Lett. 61, 320 (2003).

62. B. Marcelis, S.J.J.M.F.Kokkelmans, G.V. Shlyapnikov, and D.S. Petrov, Phys. Rev. A 77, 032707 (2008).

63. A. Delfino, T. Frederico, M.S. Hussein, and L. Tomio, Phys. Rev. C 61, 051301 (2000).

64. M. T. Yamashita, T. Frederico, and L. Tomio, Phys. Lett. B 670, 49 (2008).

65. L.M. Delves, Phys. Rev. 118, 1318 (1960).

66. W.T.H. van Oers and J.D. Seagrave, Phys. Lett. 24B, 562 (1967).

67. A. S. Reiner, Phys. Lett. 28B, 387 (1969).

68. J.S. Whiting and M.G. Fuda, Phys. Rev. C 14, $18(1976)$.

69. B. A. Girard and M.G. Fuda, Phys. Rev. C 19, 579 (1979).

70. S. K. Adhikari and J. R. A. Torreão, Phys. Lett. 119B, 245 (1982); Phys. Lett. 132B 257 (1983); S. K. Adhikari, Phys. Rev. C 30, 31 (1984).

71. I. V. Simenog, A. I. Sitnichenko and D. V. Shapoval, Sov. J. Nucl. Phys. 45, 37 (1987).

72. S. K. Adhikari, A. C. Fonseca, and L. Tomio, Phys. Rev. C 26, 77 (1982); S. K. Adhikari and L. Tomio, Phys. Rev. C 26, 83 (1982); S. K. Adhikari, L. Tomio and A. C. Fonseca, Phys. Rev. C 27, 1826 (1983).

73. A. Bulgac, Phys. Rev. Lett. 89, 050402 (2002); B.J. Cusack, T.J. Alexander, E.A. Ostrovskaya, and Y.S. Kivshar, Phys. Rev. A 65, 013609 (2002); E. Braaten, H.-W. Hammer, and T. Mehen, Phys. Rev. Lett. 88, 040401 (2002); E. Braaten, H.-W. Hammer, and M. Kusunoki, Phys. Rev. Lett. 90, 170402 (2003). 